\title{
含三氟甲基苯基的三唑化合物的合成、晶体结构及杀菌活性研究
}

\author{
余陈升 $a$ 王 翰 $a$ 闵莉静 $b$ 韩 亮 $a$ 史建俊 ${ }^{*}, c$ 刘幸海 $*, a$ \\ ( ${ }^{a}$ 浙江工业大学化学工程学院 杭州 310014) \\ $(b$ 湖州师范学院生命科学学院 浙江省媒介生物学与病原控制重点实验室 浙江湖州 313000) \\ ( ${ }^{c}$ 黄山学院化学化工学院 安徽黄山 245041)
}

\begin{abstract}
摘要 为了寻找高杀菌活性的农药先导化合物，以烯效唑为先导化合物，设计合成了一系列含三氟甲基苯基的三坐类 化合物, 其结构经 ${ }^{1} \mathrm{H}$ NMR, ${ }^{13} \mathrm{C}$ NMR 和 HRMS 进行了确证, 且(E)-1-苯基-2-(1H-1,2,4-三唑-1-基)-3-(4-三氟甲基苯基) 烯丙基苯甲酸酯(5a) 经过 X 单晶衍射认证. 初步的生物活性测定结果表明, 在 $50 \mu \mathrm{g} / \mathrm{mL}$ 的浓度下, 目标化合物对所测 菌类均有一定的杀菌活性, 其中对油菜菌核病菌和水稻纹枯病菌的杀菌效果最佳.
\end{abstract}

关键词 合成; 晶体结构; 三唑类杀菌剂; 分子对接

\section{Synthesis, Cyrstal Structure and Fungicidal Activity of New Triazole Compounds Containing Trifluoromethylphenyl Moiety}

\author{
Yu, Chensheng ${ }^{a} \quad$ Wang, Han $^{a} \quad$ Min, Lijing ${ }^{b} \quad$ Han, Liang ${ }^{a}$ \\ Shi, Jianjun *,c Liu, Xinghai*,a \\ ( ${ }^{a}$ College of Chemical Engineering, Zhejiang University of Technology, Hangzhou 310014) \\ $\left({ }^{b}\right.$ Key Laboratory of Vector Biology and Pathogen Control of Zhejiang Province, College of Life Science, \\ Huzhou University, Huzhou, Zhejiang 313000) \\ ( ${ }^{c}$ College of Chemistry \& Chemical Engineering, Huangshan University, Huangshan, Anhui 245041)
}

\begin{abstract}
In order to find new lead compounds with high activity, a series of 1,2,4-triazole compounds were designed and synthesized by using Uniconazole as temple compound. Their structures were confirmed by ${ }^{1} \mathrm{H}$ NMR, ${ }^{13} \mathrm{C}$ NMR and HRMS spectra, and the crystal structure of (E)-1-phenyl-2-(1H-1,2,4-triazol-1-yl)-3-(4-(trifluoromethyl)phenyl)allyl-benzoate (5a) was determined by X-ray single crystal diffraction. The bioassay data showed that some of target compounds 5 exhibited good activity ( $>50 \%$ ) against Sclerotinia sclerotiorum and Rhizoctonia solanimore at $50 \mu \mathrm{g} / \mathrm{mL}$.

Keywords synthesis; crystal structure; triazole fungicides; molecular docking
\end{abstract}

\section{Introduction}

With the development of modern agriculture and the improvement of environmental protection awareness pesticide discovery had improved and evolved from wholesale elimination to biological regulation. ${ }^{[1]}$ Since heterocyclic compounds have wide biological activity, low toxicity, high efficiency, and structural diversity, they have become an area of focus in drug discovery..$^{[2-4]}$ Among heterocyclic compounds, 1,2,4-triazole is a classic five-membering heterocycle used in medicinal and pesticidal industry, including herbicidal. ${ }^{[5-6]}$ antibacterial, ${ }^{[7]}$ antifungal, ${ }^{[8]}$ insecti- cidal, ${ }^{[9-10]}$ anti-inflammatory, ${ }^{[11-12]}$ antiviral, ${ }^{[13]}$ anti-influen$\mathrm{za}{ }^{[14]}$ anticancer, ${ }^{[15]}$ antioxidative, ${ }^{[16]}$ and anticonvulsant. ${ }^{[17-18]}$ For example, the earliest triazole pesticide, triamiphos, was synthesized by N.V-Pnlipn Dnphan company in the early 1960s. After that, many 1,2,4-triazole antifungals were discovered, such as triadimenol, triadimonol diniconazole and uniconazole.

In our previous work triazole compounds $(Z$ configuration) were synthesized and exhibited certain fungicidal activity. ${ }^{[19]}$ Since fluorine atoms have special properties of bioactive compounds, such as metabolic stability, better bioavailability, ${ }^{[20-21]}$ and always possessed excellent bio-

\footnotetext{
* Corresponding authors. E-mail: xhliu@zjut.edu.cn; sjj@hsu.edu.cn

Received June 11, 2021; revised July 9, 2021; published online July 23, 2021.

Project supported by the Zhejiang Provincial Natural Science Foundation (Nos. LY19C140002, LY19B020009), and the Three Win Material Company (No. KYY-HX-20200746).

浙江省自然科学基金(Nos. LY19C140002, LY19B020009)、浙江三赢新材料有限公司(No. KYY-HX-20200746)资助项目.
} 
logical activity, ${ }^{[22]}$ they were often introduced into the structures of medicines or pesticides. ${ }^{[23-24]}$ In order to discover new lead compounds, uniconazole was used as lead compound, $t$ - $\mathrm{Bu}$ group was used instead of phenyl ring, hydroxyl group was esterified, the chlorine atom was replaced by $\mathrm{CF}_{3}$ atom on the phenyl ring, and a series of 1,2,4-triazole compounds ( $E$ configuration) were designed and synthesized (Figure 1). Some of the compounds showed good fungicidal activity.

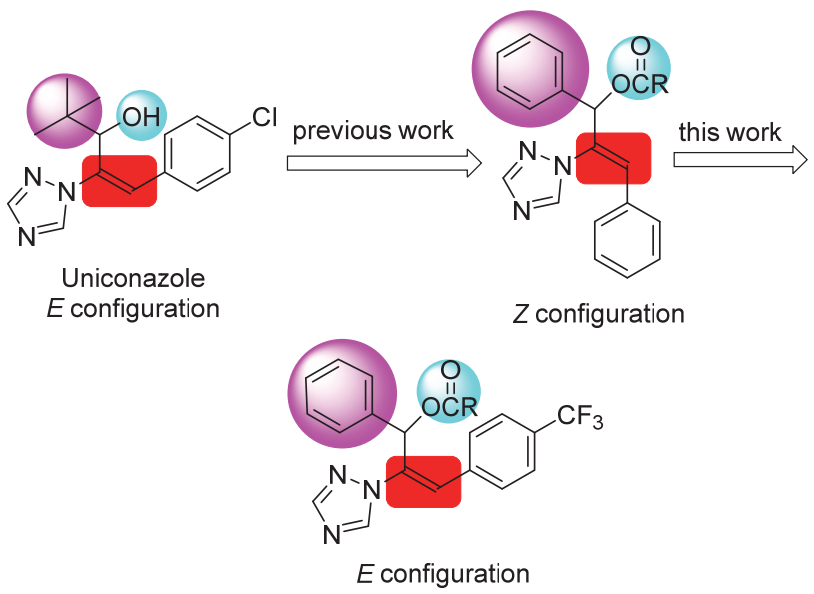

Figure 1 Design strategy of the title compound $\mathbf{5}$

\section{Results and discussion}

\subsection{Synthesis and spectra of target triazole com- pounds}

The synthetic route of 1,2,4-triazole compounds is shown in Scheme 1. $\alpha$-Bromoacetophenone 1 reacted with 1,2,4-triazole under base conditions to give 1-phenyl-2(1H-1,2,4-triazol-1-yl)ethan-1-one (2). Intermediate 2 mixed with 4-(trifluoromethyl)benzaldehyde according to Knoevenagel condensation to give 1-phenyl-2-(1H-1,2,4triazol-1-yl)-3-(4-(trifluoromethyl)phenyl)prop-2-en-1-one (3). In this process Dean-Stark trap was used, but a molecular sieve could also be used in place of Dean-Stark trap. Intermediate 3 reduced by $\mathrm{NaBH}_{4}$ gave 1-phenyl-2-(1H1,2,4-triazol-1-yl)-3-(4-(trifluoromethyl)phenyl)prop-2-en1-ol (4). Intermediate 4 was then esterified with different acid to prepare target compounds $\mathbf{5 a} \sim \mathbf{5} \mathbf{j}$.

\subsection{Crystal structure of target compound $\mathbf{5 a}$}

The target compound $\mathbf{5}$ has two configurations: $Z$ or $E$. In order to determine the configuration, the target compound 5a was analyzed by X-ray crystal diffraction. Some representative bond angles and bond distances of compound 5a are listed in supporting information. The molecular structure and packing diagram of compound 5a are illustrated in Figure 2.

The general bond angles and bond lengths of ring systems (1,2,4-triazole ring and phenyl ring) were in normal ranges. For the 1,2,4-triazole ring, $\mathrm{N}(2)-\mathrm{C}(17)[0.1313(2)$ $\mathrm{nm}], \mathrm{N}(1)-\mathrm{C}(16)[0.1354(2) \mathrm{nm}]$ and $\mathrm{N}(1)-\mathrm{C}(15)$ $[0.1433(2) \mathrm{nm}]$ bonds were a little longer than the normal $\mathrm{C}=\mathrm{N}(0.127 \mathrm{~nm})$, which indicated electron delocalized on<smiles></smiles>

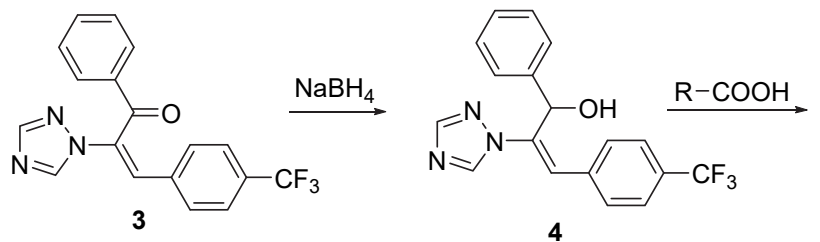<smiles>[R]C(=O)OC(/C(=C/c1ccc(C(F)(F)F)cc1)n1cncn1)c1ccccc1</smiles>

5

5a: $\mathrm{R}=\mathrm{Ph} ; \mathbf{5 b}: \mathrm{R}=2-\mathrm{MeC}_{6} \mathrm{H}_{4} ; \mathbf{5 c}: \mathrm{R}=2-\mathrm{FC}_{6} \mathrm{H}_{4} ; \mathbf{5 d}: \mathrm{R}=4-\mathrm{BuC}_{6} \mathrm{H}_{4}$ 5e: $\mathrm{R}=3-\mathrm{ClC}_{6} \mathrm{H}_{4} ; \mathbf{5 f}: \mathrm{R}=4-\mathrm{PrC}_{6} \mathrm{H}_{4} ; \mathbf{5 g}: \mathrm{R}=4-\mathrm{MeOC}_{6} \mathrm{H}_{4} ; \mathbf{5 h}: \mathrm{R}=$ $2,4-\mathrm{Cl}_{2} \mathrm{C}_{6} \mathrm{H}_{3} ; 5 \mathrm{i}: \mathrm{R}=3-\mathrm{O}_{2} \mathrm{NC}_{6} \mathrm{H}_{4} ; \mathbf{5 j}: \mathrm{R}=2,3-\mathrm{Cl}_{2} \mathrm{C}_{6} \mathrm{H}_{3}$

Scheme 1 Synthetic route of the target compound 5
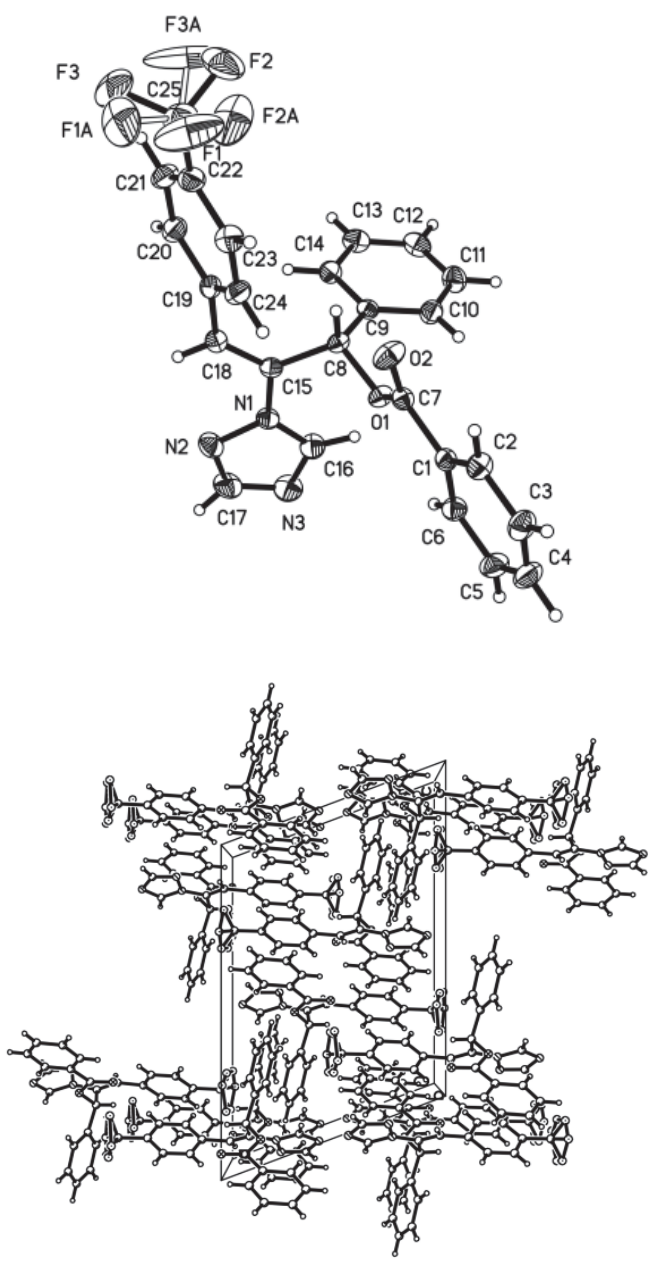

Figure 2 Molecular structure and packing diagram of the title compound $\mathbf{5 a}$

1,2,4-triazole ring. The bond length of $\mathrm{C}(7)-\mathrm{O}(2)$ is 
$0.12064(19) \mathrm{nm}$, which is according to $\mathrm{C}=\mathrm{O}$ group. The torsion angles of $\mathrm{C}(1)-\mathrm{C}(7)-\mathrm{O}(1)-\mathrm{C}(8)$ and $\mathrm{O}(2)-$ $\mathrm{C}(7)-\mathrm{O}(1)-\mathrm{C}(8)$ were $177.98(11)^{\circ}$ and $-1.9(2)^{\circ}$, respectively, which indicated that the phenyl ring and the ester group were in the same plane. As shown in Figure 2, the phenyl ring $[\mathrm{C}(9) \sim \mathrm{C}(11)]$ is vertical with 1,2,4-triazole ring $[\mathrm{N}(1), \mathrm{N}(2), \mathrm{C}(1), \mathrm{N}(3), \mathrm{C}(16)]$, with a dihedral angle $(\theta)$ of $89.2^{\circ}$ with plane equation $-5.322 x+5.891$ $y+14.146 z=-4.6004$ and $-3.253 x-5.865 y+14.404$ $z=-0.9652$, respectively. On the other hand, the two phenyl rings $[\mathrm{C}(1) \sim \mathrm{C}(6)$ and $\mathrm{C}(19) \sim \mathrm{C}(24)]$ are nearly parallel with a dihedral angle $(\theta)$ of $1.2^{\circ}$. The other rings have dihedral angles $(\theta)$ of $65.0^{\circ}, 74.8^{\circ}, 64.8^{\circ}$, and $76.0^{\circ}$, respectively. There are also two intermolecular face-toface $\pi-\pi$ stackings between the two benzene rings $[\mathrm{C}(1) \sim$ $\mathrm{C}(6)$ and $\mathrm{C}(19) \sim \mathrm{C}(24)$ ] in the crystal. It is worth noting that the two molecules of benzene ring-benzene rings are centrosymmetric: the centroid distance of benzene ring-benzene ring is $0.3837 \mathrm{~nm}$. These $\pi-\pi$ stackings formed infinite one-dimensional chain structures. More importantly, $\mathrm{C}(19)$ and $\mathrm{N}(1)$ in the target compound are on the opposite side of $\mathrm{C}(15)$ and $\mathrm{C}(18)$, therefore it can be concluded that the target compound $\mathbf{5 a}$ is $E$ configuration.

\subsection{Fungicidal activity of the compound}

The results of antifungal activity against ten different fungi are shown in Table 1. Most of the title compounds exhibited moderate activity $(>50 \%)$ against $S$. sclerotiorum at $50 \mu \mathrm{g} / \mathrm{mL}$, except compounds $\mathbf{5 d}$ and $\mathbf{5 j}$. In addition to this, the target compound also showed good antifungal activity against $R$. solani, except for compound $\mathbf{5} \mathbf{j}$, which performed the same as the positive control fluxapyroxad. Interestingly, the compound $\mathbf{5 g}$ possessed good activity against $G$. zeae, which is higher than that of the positive control fluxapyroxad. It is worth noting that all target compounds $\mathbf{5}$ exhibit moderate antifungal activity against $P$. capsici, which are better than the positive control. For example, compounds 5a (42.9\%) and 5c (42.9\%) possessed moderate inhibitory activity against $P$. capsici, which were better than the positive fluxapyroxad (16.7\%). In addition, the activity of $\mathbf{5 b}$ against $P$. infestans was also higher than that of the control group. Unfortunately, the target compounds had nearly no active activity against $F$. oxysporum, $C$. arachidicola, $P$. piricola. The synthesis of these new fungicides provides a basis for preparing lead compounds with higher activity in the future.

\subsection{Molecular docking}

The target of triazole fungicides is sterol $14 \alpha$-demethylase (CYP51), therefore molecular docking between compound 5c and sterol $14 \alpha$-demethylase (CYP51) ${ }^{[25]}$ was performed according to the reference method and carried out by using Discovery Studio 2.5 software. The crystal structure of sterol 14 $\alpha$-demethylase (CYP51) (PDB:5JLC) was downloaded from the PDB bank ((http://www. rcsb.org). As shown in Figure 3, compound $\mathbf{5 c}$ is wellmatched to the active pocket of sterol $14 \alpha$-demethylase (CYP51). The compound 5c was discovered to constitute a bidentate combination with the $\mathrm{Fe}^{2+}$. The benzene ring formed $\pi-\pi$ stacking interaction with pyrrole ring in the porphyrin. It was observed that the distance between the benzene ring and pyrrole ring was $0.57 \mathrm{~nm}$. Meanwhile, there is a hydrogen bond between the $\mathrm{CF}_{3}$ and the amino acid residue Val 462 with the distance of $0.21 \mathrm{~nm}$.

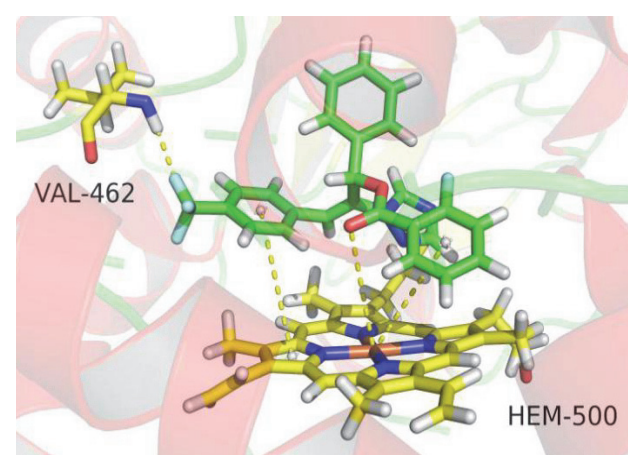

Figure 3 Molecular docking diagram of compound $\mathbf{5 c}$ with target sterol 14 $\alpha$-demethylase (CYP51)

Table 1 Inhibition rate (\%) of target compounds $\mathbf{5 a} \sim \mathbf{5 j}$ at $50 \mu \mathrm{g} / \mathrm{mL}^{a}$

\begin{tabular}{ccccccccccc}
\hline Compd. & $A S$ & $G Z$ & $P I$ & $P C$ & $S S$ & $B C$ & $R S$ & $F O$ & $C A$ & $P P$ \\
\hline $\mathbf{5 a}$ & 15.4 & 30.4 & 25.0 & 42.9 & 53.6 & 33.3 & 54.8 & 13.6 & 8.3 & 8.3 \\
$\mathbf{5 b}$ & 7.7 & 30.4 & 31.3 & 39.3 & 62.5 & 9.5 & 42.9 & 4.5 & 16.7 & 8.3 \\
$\mathbf{5 c}$ & 11.5 & 30.4 & 25.0 & 42.9 & 67.9 & 33.3 & 50.0 & 4.5 & 8.3 & 8.3 \\
$\mathbf{5 d}$ & 11.5 & 8.7 & 12.5 & 25.0 & 26.8 & 33.3 & 31.0 & 9.1 & 8.3 & 8.3 \\
$\mathbf{5 e}$ & 7.7 & 17.4 & 6.3 & 25.0 & 53.6 & 23.8 & 38.1 & 4.5 & 16.7 & 25.0 \\
$\mathbf{5 f}$ & 15.4 & 17.4 & 6.3 & 32.1 & 53.6 & 38.1 & 50.0 & 9.1 & 25.0 & 33.3 \\
$\mathbf{5 g}$ & 38.5 & 52.2 & 18.8 & 39.3 & 58.9 & 38.1 & 66.7 & 4.5 & 25.0 & 33.3 \\
$\mathbf{5 h}$ & 23.1 & 17.4 & 12.5 & 25.0 & 50.0 & 33.3 & 38.1 & 4.5 & 16.7 & 8.3 \\
$\mathbf{5 i}$ & 23.1 & 21.7 & 12.5 & 35.7 & 57.1 & 33.3 & 38.1 & 13.6 & 25.0 & 8.3 \\
$\mathbf{5 j}$ & 11.5 & 8.7 & 25.0 & 35.7 & 35.7 & 23.8 & 14.3 & 13.6 & 8.3 & 8.3 \\
CK & 0 & 0 & 0 & 0 & 0 & 0 & 0 & 0 & 0 & 0 \\
Fluxapyroxad & 88.9 & 28.6 & 27.3 & 16.7 & 96.4 & 63.6 & 88.4 & 100 & 44.4 & 84.6 \\
\hline
\end{tabular}

a Alternaria solani (AS), Gibberella zeae (GZ), Phytophthora infestans (PI), Phytophthora capsici (PC), Sclerotinia sclerotiorum (SS), Botrytis cinerea (BC), Rhizoctonia solani (RS), Fusarium oxysporum (FO), Cercospora arachidicola (CA), Physalospora piricola $(P P)$. 


\section{Conclusions}

In conclusion, a series of triazole compounds containing trifluoromethyl phenyl groups were designed and synthesized through substitution, condensation, reduction, and esterification reactions. The bioassay showed that some compounds exhibited good fungicidal activity at $50 \mu \mathrm{g} / \mathrm{mL}$. The structure-activity relationship was studied using molecular docking.

\section{Experimental section}

\subsection{Instruments and reagents}

Melting points were determined using an X-4 digital microscopic melting point detector (Taike, Beijing, China) and uncorrected. ${ }^{1} \mathrm{H}$ NMR spectra were measured on a Bruker AV-500 instruments using TMS as an internal standard and $\mathrm{CDCl}_{3}$ as the solvent. HRMS was determined on an Agilent 1200RRLC-6520 instrument. All the reagents are of analytical grade or freshly prepared before use. Analytical TLC (Huanghai, Qingdao, China) was performed on silica gel $\mathrm{GF}_{254}$.

\subsection{Synthesis}

4.2.1 Synthesis of 1-phenyl-2-(1H-1,2,4-triazol-1-yl)ethan-1-one (2)

To a solution of $\alpha$-bromoacetophenone (19.90 g, 0.10 $\mathrm{mol})$ and 1,2,4-triazole $(7.60 \mathrm{~g}, 0.11 \mathrm{~mol})$ in acetone $(150$ $\mathrm{mL}), \mathrm{NEt}_{3}(11.13 \mathrm{~g} 0.11 \mathrm{~mol})$ was added dropwise at $0{ }^{\circ} \mathrm{C}$. The mixture was stirred for $60 \mathrm{~min}$ at $0{ }^{\circ} \mathrm{C}$ and then stirred for another $60 \mathrm{~min}$ at room temperature. After the reaction, the mixture was filtered and acetone evaporated. Product by recrystallization with isopropanol formed as white crystals, yield $82.71 \%$. m.p. $106 \sim 107{ }^{\circ} \mathrm{C}$ (lit. ${ }^{[26]}$ $\left.113 \sim 115{ }^{\circ} \mathrm{C}\right) ;{ }^{1} \mathrm{H} \mathrm{NMR}\left(500 \mathrm{MHz}, \mathrm{CDCl}_{3}\right) \delta: 8.21(\mathrm{~s}, 1 \mathrm{H}$, triazole-H), $8.16(\mathrm{~s}, 1 \mathrm{H}$, triazole-H), $7.87 \sim 7.81(\mathrm{~m}, 2 \mathrm{H}$, ArH), $7.68 \sim 7.57$ (m, 2H, ArH), $7.55 \sim 7.48$ (m, 2H, ArH), $7.35 \sim 7.23(\mathrm{~m}, 3 \mathrm{H}, \mathrm{ArH}), 6.94(\mathrm{~d}, J=1.0 \mathrm{~Hz}, 1 \mathrm{H},=\mathrm{CH})$.

4.2.2 Synthesis of 1-phenyl-2-(1H-1,2,4-triazol-1-yl)3-(4-(trifluoromethyl)phenyl)prop-2-en-1-one (3)

To a solution of intermediate $2(5.62 \mathrm{~g}, 30 \mathrm{mmol})$ in toluene $(30 \mathrm{~mL}), p$-trifluoromethylbenzaldehyde $(5.75 \mathrm{~g}$, $33 \mathrm{mmol}$ ) was added. Piperidine was dropwise to mixture and refluxed for $7 \mathrm{~h}$. Then the solvent was removed. The residue was purified by column chromatography (petroleum ether/ethyl acetate (PE/EA), $V / V=1 / 1$ ) to obtain a pale yellow solid 3, yield 55.6\%. m.p. 196 197 ${ }^{\circ} \mathrm{C} ;{ }^{1} \mathrm{H}$ NMR $\left(500 \mathrm{MHz}, \mathrm{CDCl}_{3}\right) \delta: 8.21(\mathrm{~s}, 1 \mathrm{H}$, triazole-H), $8.16(\mathrm{~s}, 1 \mathrm{H}$, triazole-H), $7.87 \sim 7.81(\mathrm{~m}, 2 \mathrm{H}, \mathrm{ArH}), 7.68 \sim 7.57(\mathrm{~m}, 2 \mathrm{H}$, ArH), $7.55 \sim 7.48(\mathrm{~m}, 2 \mathrm{H}, \mathrm{ArH}), 7.35 \sim 7.23(\mathrm{~m}, 3 \mathrm{H}, \mathrm{ArH})$, $6.94(\mathrm{~d}, J=1.0 \mathrm{~Hz}, 1 \mathrm{H},=\mathrm{CH})$; HRMS (ESI) calcd for $\mathrm{C}_{18} \mathrm{H}_{13} \mathrm{~F}_{3} \mathrm{~N}_{3} \mathrm{O}[\mathrm{M}+\mathrm{H}]^{+}$344.1005, found 344.1001.

4.2.3 Synthesis of 1-phenyl-2-(1H-1,2,4-triazol-1-yl)3-(4-(trifluoromethyl)phenyl)prop-2-en-1-ol (4)

To a solution of intermediate $3(2.40 \mathrm{~g}, 7.00 \mathrm{mmol})$ and PEG $_{600}(0.50 \mathrm{~g}, 0.83 \mathrm{mmol})$ in methanol $(15 \mathrm{~mL})$, sodium borohydride $(0.42 \mathrm{~g}, 11.0 \mathrm{mmol})$ was added in portions with stirring at $0{ }^{\circ} \mathrm{C}$. The mixture continued to be stirred for $4 \mathrm{~h}$ at $0{ }^{\circ} \mathrm{C}$. After the reaction was completed, the methanol was evaporated. The residue was poured into water $(10 \mathrm{~mL})$ containing $5 \mathrm{~mL}$ of dilute $\mathrm{HCl}$. Then, it was washed by $\mathrm{CH}_{2} \mathrm{Cl}_{2}(20 \mathrm{~mL} \times 3)$, the organic layer was dried with $\mathrm{Na}_{2} \mathrm{SO}_{4}$ and evaporated to give a yellow oil, yield $91.20 \%$. ${ }^{1} \mathrm{H}$ NMR $\left(500 \mathrm{MHz}, \mathrm{CDCl}_{3}\right) \delta: 7.95(\mathrm{~s}, 1 \mathrm{H}$, triazole-H), 7.42 $(\mathrm{s}, 1 \mathrm{H}$, triazole-H), $7.30 \sim 7.24(\mathrm{~m}, 4 \mathrm{H}, \mathrm{ArH}), 7.23 \sim 7.14$ (m, 4H, ArH), $6.93(\mathrm{~d}, J=1.0 \mathrm{~Hz}, 1 \mathrm{H},=\mathrm{CH}), 6.80(\mathrm{~s}, 1 \mathrm{H}$, ArH), 5.65 (s, 1H, CH), $5.12(\mathrm{~s}, 1 \mathrm{H}, \mathrm{OH})$; HRMS (ESI) calcd for $\mathrm{C}_{18} \mathrm{H}_{15} \mathrm{~F}_{3} \mathrm{~N}_{3} \mathrm{O}[\mathrm{M}+\mathrm{H}]{ }^{+}$346.1162, found 346.1157 .

\subsubsection{General synthesis of target compound 5}

To a solution of intermediate $4(0.25 \mathrm{~g}, 0.72 \mathrm{mmol})$, 4dimethylaminopyridine oxide (DMAP) $(0.09 \mathrm{~g}, \quad 0.72$ mmol), 1-ethyl-3-(3-dimethylaminopropyl)carbodiimide hydrochloride (EDCI) $(0.28 \mathrm{~g}, 1.44 \mathrm{mmol})$ in $\mathrm{CH}_{2} \mathrm{Cl}_{2}$ (5 $\mathrm{mL})$, substituted benzoic acid $(0.80 \mathrm{mmol})$ was added. The mixture was stirred at room temperature for $4 \mathrm{~h}$. Then the solvent was removed and purified by column chromatography $\left[V_{\mathrm{EA}} / V_{\mathrm{PE}}=1 / 2\right]$ to obtain the target compound 5 .

(E)-1-Phenyl-2-(1H-1,2,4-triazol-1-yl)-3-(4-(trifluoromethyl)phenyl)allyl benzoate (5a): Yellow solid, yield $46.37 \%$. m.p. $148 \sim 150{ }^{\circ} \mathrm{C} ;{ }^{1} \mathrm{H}$ NMR $\left(500 \mathrm{MHz}, \mathrm{CDCl}_{3}\right) \delta$ : $8.10(\mathrm{~s}, 1 \mathrm{H}$, triazole-H), $8.09 \sim 8.07(\mathrm{~m}, 2 \mathrm{H}, \mathrm{ArH}), 7.67(\mathrm{~s}$, $1 \mathrm{H}$, triazole-H), $7.59(\mathrm{t}, J=7.5 \mathrm{~Hz}, 1 \mathrm{H}, \mathrm{ArH}), 7.49 \sim 7.42$ $(\mathrm{m}, 6 \mathrm{H}, \mathrm{ArH}), 7.40 \sim 7.33(\mathrm{~m}, 3 \mathrm{H}, \mathrm{ArH}), 7.04(\mathrm{~s}, 1 \mathrm{H}, \mathrm{CH})$, $6.99(\mathrm{~s}, 1 \mathrm{H},=\mathrm{CH}), 6.96(\mathrm{~d}, J=8.3 \mathrm{~Hz}, 2 \mathrm{H}, \mathrm{ArH}) ;{ }^{13} \mathrm{C}$ NMR $\left(400 \mathrm{MHz}, \mathrm{CDCl}_{3}\right) \delta: 164.95,151.51,143.26$, $136.99,135.66,134.14,133.87,129.78,129.25,129.14$, $128.85,128.74,125.88,125.85,125.81,125.77,125.56$, 70.55. HRMS (ESI) calcd for $\mathrm{C}_{25} \mathrm{H}_{19} \mathrm{~F}_{3} \mathrm{~N}_{3} \mathrm{O}_{2}[\mathrm{M}+\mathrm{H}]$ 450.1424 , found 450.1423 .

(E)-1-Phenyl-2-(1H-1,2,4-triazol-1-yl)-3-(4-(trifluoromethyl)phenyl)allyl 2-methylbenzoate (5b): Yellow solid, yield 58.38\%. m.p. $96 \sim 98{ }^{\circ} \mathrm{C} ;{ }^{1} \mathrm{H}$ NMR $(500 \mathrm{MHz}$, $\left.\mathrm{CDCl}_{3}\right) \delta: 8.10(\mathrm{~s}, 1 \mathrm{H}$, triazole-H), $8.01 \sim 7.94(\mathrm{~m}, 1 \mathrm{H}$, ArH), $7.65(\mathrm{~s}, 1 \mathrm{H}$, triazole-H), $7.48 \sim 7.42(\mathrm{~m}, 5 \mathrm{H}, \mathrm{ArH})$, $7.39 \sim 7.33(\mathrm{~m}, 3 \mathrm{H}, \mathrm{ArH}), 7.26(\mathrm{t}, J=7.4 \mathrm{~Hz}, 2 \mathrm{H}, \mathrm{ArH})$, $7.03(\mathrm{~s}, 1 \mathrm{H}, \mathrm{CH}), 6.96(\mathrm{~s}, 2 \mathrm{H}, \mathrm{ArH}), 6.94(\mathrm{~s}, 1 \mathrm{H},=\mathrm{CH})$, $2.57(\mathrm{~s}, 3 \mathrm{H}, \mathrm{CH} 3) ;{ }^{13} \mathrm{C} \mathrm{NMR}\left(400 \mathrm{MHz}, \mathrm{CDCl}_{3}\right) \delta: 165.47$, $152.75,144.39,140.96,136.37,135.81,132.60,131.91$, $130.52,129.06,128.94,128.81,128.45,127.39,126.95$, 125.91, 125.70, 75.69, 21.73. HRMS (ESI) calcd for $\mathrm{C}_{26} \mathrm{H}_{21} \mathrm{~F}_{3} \mathrm{~N}_{3} \mathrm{O}_{2}[\mathrm{M}+\mathrm{H}]^{+}$464.1580, found 464.1577.

(E)-1-Phenyl-2-(1H-1,2,4-triazol-1-yl)-3-(4-(trifluoromethyl)phenyl)allyl 2-fluorobenzoate (5c): Yellow solid, yield 72.35\%. m.p. $93 \sim 95{ }^{\circ} \mathrm{C} ;{ }^{1} \mathrm{H}$ NMR $(500 \mathrm{MHz}$, $\left.\mathrm{CDCl}_{3}\right) \delta: 8.08(\mathrm{~s}, 1 \mathrm{H}$, triazole-H), $8.01 \sim 7.94(\mathrm{~m}, 1 \mathrm{H}$, ArH), $7.69(\mathrm{~s}, 1 \mathrm{H}$, triazole-H), $7.61 \sim 7.52(\mathrm{~m}, 1 \mathrm{H}, \mathrm{ArH})$, $7.48 \sim 7.42(\mathrm{~m}, 4 \mathrm{H}, \mathrm{ArH}), 7.38 \sim 7.32(\mathrm{~m}, 3 \mathrm{H}, \mathrm{ArH}), 7.23(\mathrm{t}$, $J=7.6 \mathrm{~Hz}, 1 \mathrm{H}, \mathrm{ArH}), 7.20 \sim 7.14(\mathrm{~m}, 1 \mathrm{H}, \mathrm{ArH}), 7.07(\mathrm{~s}$, $1 \mathrm{H}, \mathrm{CH}), 7.04(\mathrm{~s}, 1 \mathrm{H},=\mathrm{CH}), 6.96(\mathrm{~d}, J=8.2 \mathrm{~Hz}, 2 \mathrm{H}$, $\mathrm{ArH}) ;{ }^{13} \mathrm{C} \mathrm{NMR}\left(400 \mathrm{MHz}, \mathrm{CDCl}_{3}\right) \delta: 163.33,162.88$, $162.85,160.75,152.73,144.47,135.94,135.86,135.51$, $135.27,135.18,132.52,130.90,130.57,129.11,128.91$, 
$128.85,127.50,126.94,125.67,124.25,117.26,117.04$, 76.28. HRMS (ESI) calcd for $\mathrm{C}_{25} \mathrm{H}_{18} \mathrm{~F}_{4} \mathrm{~N}_{3} \mathrm{O}_{2}[\mathrm{M}+\mathrm{H}]^{+}$ 468.1330, found 468.1327.

(E)-1-Phenyl-2-(1H-1,2,4-triazol-1-yl)-3-(4-(trifluoromethyl)phenyl)allyl 4-(tert-butyl)benzoate (5d): White solid, yield 55.16\%. m.p. 95 96 ${ }^{\circ} \mathrm{C} ;{ }^{1} \mathrm{H}$ NMR $(500 \mathrm{MHz}$, $\left.\mathrm{CDCl}_{3}\right) \delta: 8.08(\mathrm{~s}, 1 \mathrm{H}$, triazole-H), $8.05 \sim 8.01(\mathrm{~m}, 2 \mathrm{H}$, $\mathrm{ArH}), 7.68(\mathrm{~s}, 1 \mathrm{H}$, triazole-H), $7.50 \sim 7.48$ (m, 2H, ArH), $7.47 \sim 7.42(\mathrm{~m}, 4 \mathrm{H}, \mathrm{ArH}), 7.38 \sim 7.32(\mathrm{~m}, 3 \mathrm{H}, \mathrm{ArH}), 7.03$ $(\mathrm{s}, 1 \mathrm{H}, \mathrm{CH}), 6.98(\mathrm{~s}, 1 \mathrm{H},=\mathrm{CH}), 6.95(\mathrm{~d}, J=8.3 \mathrm{~Hz}, 2 \mathrm{H}$, $\mathrm{ArH}), 1.34$ (s, 9H, $t$-Bu); ${ }^{13} \mathrm{C}$ NMR (400 MHz, $\left.\mathrm{CDCl}_{3}\right) \delta$ : $164.85,157.45,152.71,144.35,136.29,135.80,129.67$, $129.02,128.89,128.83,127.62,126.84,126.46,125.62$, 75.66, 35.16, 31.04. HRMS (ESI) calcd for $\mathrm{C}_{29} \mathrm{H}_{27} \mathrm{~F}_{3} \mathrm{~N}_{3} \mathrm{O}_{2}$ $[\mathrm{M}+\mathrm{H}]^{+}$506.2050, found 506.2061.

(E)-1-Phenyl-2-(1H-1,2,4-triazol-1-yl)-3-(4-(trifluoromethyl)phenyl)allyl 3-chlorobenzoate (5e): Yellow solid, yield 48.75\%. m.p. $148 \sim 151{ }^{\circ} \mathrm{C}$; ${ }^{1} \mathrm{H}$ NMR $(500 \mathrm{MHz}$, $\left.\mathrm{CDCl}_{3}\right) \delta: 8.10(\mathrm{~s}, 1 \mathrm{H}$, triazole-H), $8.04(\mathrm{t}, J=1.8 \mathrm{~Hz}, 1 \mathrm{H}$, ArH $), 7.98 \sim 7.94(\mathrm{~m}, 1 \mathrm{H}, \mathrm{ArH}), 7.64(\mathrm{~s}, 1 \mathrm{H}$, triazole-H), $7.59 \sim 7.55(\mathrm{~m}, 1 \mathrm{H}, \mathrm{ArH}), 7.46(\mathrm{~d}, J=8.3 \mathrm{~Hz}, 2 \mathrm{H}, \mathrm{ArH})$, $7.43 \sim 7.36(\mathrm{~m}, 6 \mathrm{H}, \mathrm{ArH}), 7.04(\mathrm{~s}, 1 \mathrm{H}, \mathrm{CH}), 6.97(\mathrm{~s}, 2 \mathrm{H}$, ArH), $6.95(\mathrm{~s}, 1 \mathrm{H},=\mathrm{CH}) ;{ }^{13} \mathrm{C}$ NMR $\left(400 \mathrm{MHz}, \mathrm{CDCl}_{3}\right) \delta$ : $163.75,152.81,144.37,135.91,135.34,134.78,133.63$, $131.03,129.95,129.76,129.26,129.01,128.83,127.90$, 127.86, 126.87, 125.73, 125.70, 76.27. HRMS (ESI) calcd for $\mathrm{C}_{25} \mathrm{H}_{18} \mathrm{ClF}_{3} \mathrm{~N}_{3} \mathrm{O}_{2}[\mathrm{M}+\mathrm{H}]^{+}$484.1034, found 484.1028 .

(E)-1-Phenyl-2-(1H-1,2,4-triazol-1-yl)-3-(4-(trifluoromethyl)phenyl)allyl 4-propylbenzoate (5f): White solid, yield 55.32\%. m.p. 99 $101{ }^{\circ} \mathrm{C} ;{ }^{1} \mathrm{H}$ NMR $(500 \mathrm{MHz}$, $\left.\mathrm{CDCl}_{3}\right) \delta: 8.10(\mathrm{~s}, 1 \mathrm{H}$, triazole-H), $8.00(\mathrm{~d}, J=8.2 \mathrm{~Hz}, 2 \mathrm{H}$, ArH), $7.72(\mathrm{~s}, 1 \mathrm{H}$, triazole-H), $7.58(\mathrm{t}, J=7.4 \mathrm{~Hz}, 2 \mathrm{H}, \mathrm{ArH})$, $7.45 \sim 7.41(\mathrm{~m}, 3 \mathrm{H}, \mathrm{ArH}), 7.40 \sim 7.32(\mathrm{~m}, 4 \mathrm{H}, \mathrm{ArH}), 7.02$ $(\mathrm{s}, 1 \mathrm{H}, \mathrm{CH}), 6.99(\mathrm{~s}, 1 \mathrm{H},=\mathrm{CH}), 6.96(\mathrm{~d}, J=8.2 \mathrm{~Hz}, 2 \mathrm{H}$, $\operatorname{ArH}), 2.65\left(\mathrm{t}, J=7.5 \mathrm{~Hz}, 2 \mathrm{H}, \mathrm{CH}_{2}\right), 1.70 \sim 1.61(\mathrm{~m}, 2 \mathrm{H}$, $\left.\mathrm{CH}_{2}\right), 0.94\left(\mathrm{t}, J=7.3 \mathrm{~Hz}, 3 \mathrm{H}, \mathrm{CH}_{3}\right) ;{ }^{13} \mathrm{C} \mathrm{NMR}(400 \mathrm{MHz}$, $\left.\mathrm{CDCl}_{3}\right) \delta: 164.94,152.60,149.17,144.36,136.23,135.76$, $133.59,130.11,129.82,129.04,128.91,128.84,128.76$, $128.43,127.71,126.85,126.71,125.69,125.65,75.69$, 38.06, 24.21, 13.71. HRMS (ESI) calcd for $\mathrm{C}_{29} \mathrm{H}_{24} \mathrm{~F}_{3} \mathrm{~N}_{3} \mathrm{O}_{2}$ $[\mathrm{M}+\mathrm{H}]^{+}$492.1893, found 492.1861.

(E)-1-Phenyl-2-(1H-1,2,4-triazol-1-yl)-3-(4-(trifluoromethyl)phenyl)allyl 4-methoxybenzoate (5g): White solid, yield 44.10\%. m.p. $93 \sim 94{ }^{\circ} \mathrm{C} ;{ }^{1} \mathrm{H}$ NMR $(500 \mathrm{MHz}$, $\left.\mathrm{CDCl}_{3}\right) \delta: 8.08(\mathrm{~s}, 1 \mathrm{H}$, triazole-H), $8.06 \sim 8.03(\mathrm{~m}, 2 \mathrm{H}$, ArH), 7.67 (s, 1H, triazole-H), $7.46(\mathrm{~d}, J=8.3 \mathrm{~Hz}, 2 \mathrm{H}$, ArH), $7.43 \sim 7.40(\mathrm{~m}, 2 \mathrm{H}, \mathrm{ArH}), 7.38 \sim 7.33$ (m, 3H, ArH), $7.00(\mathrm{~s}, 1 \mathrm{H}, \mathrm{CH}), 6.98(\mathrm{~s}, 1 \mathrm{H},=\mathrm{CH}), 6.97 \sim 6.94(\mathrm{~m}, 4 \mathrm{H}$, $\mathrm{ArH}), 3.86\left(\mathrm{~s}, 3 \mathrm{H}, \mathrm{OCH}_{3}\right) ;{ }^{13} \mathrm{C} \mathrm{NMR}\left(400 \mathrm{MHz}, \mathrm{CDCl}_{3}\right) \delta$ : $164.57,163.89,152.69,144.33,136.34,135.88,131.87$, $129.00,128.89,128.83,127.59,126.83,125.67,121.58$, 113.89, 75.61, 55.48. HRMS (ESI) calcd for $\mathrm{C}_{27} \mathrm{H}_{20} \mathrm{~F}_{3} \mathrm{~N}_{3} \mathrm{O}_{3}$ $[\mathrm{M}+\mathrm{H}]^{+}$480.1530, found 480.1530 .

(E)-1-Phenyl-2-(1H-1,2,4-triazol-1-yl)-3-(4-(trifluoromethyl)phenyl)allyl 2,4-dichlorobenzoate (5h): Yellow solid, yield 66.16\%. m.p. 82 84 ${ }^{\circ} \mathrm{C}$; ${ }^{1} \mathrm{H}$ NMR $(500 \mathrm{MHz}$, $\left.\mathrm{CDCl}_{3}\right) \delta: 8.10(\mathrm{~s}, 1 \mathrm{H}$, triazole-H), $7.85(\mathrm{~d}, J=8.5 \mathrm{~Hz}, 1 \mathrm{H}$,
ArH), $7.63(\mathrm{~s}, 1 \mathrm{H}$, triazole-H), $7.49 \sim 7.45$ (m, 3H, ArH), $7.39 \sim 7.35(\mathrm{~m}, 4 \mathrm{H}, \mathrm{ArH}), 7.34 \sim 7.31(\mathrm{~m}, 2 \mathrm{H}, \mathrm{ArH}), 7.04$ $(\mathrm{s}, 1 \mathrm{H}, \mathrm{CH}), 7.01(\mathrm{~s}, 1 \mathrm{H},=\mathrm{CH}), 6.95(\mathrm{~d}, J=8.2 \mathrm{~Hz}, 2 \mathrm{H}$, $\mathrm{ArH}) ;{ }^{13} \mathrm{C} \mathrm{NMR}\left(400 \mathrm{MHz}, \mathrm{CDCl}_{3}\right) \delta: 163.65,163.15$, $152.79,150.58,144.47,143.80,139.08,139.03,135.65$, $135.37,135.24,135.13,135.06,133.79,133.01,132.89$, $131.25,131.22,129.27,129.14,128.99,128.81,128.35$, 128.02 , 127.76, 127.28, 127.02, 125.71, 124.79, 75.88. HRMS (ESI) calcd for $\mathrm{C}_{25} \mathrm{H}_{17} \mathrm{Cl}_{2} \mathrm{~F}_{3} \mathrm{~N}_{3} \mathrm{O}_{2}[\mathrm{M}+\mathrm{H}]$ 518.0644, found 518.0717.

(E)-1-Phenyl-2-(1H-1,2,4-triazol-1-yl)-3-(4-(trifluoromethyl)phenyl)allyl 3-nitrobenzoate (5i): White solid, yield $23.17 \%$. m.p. $133 \sim 135{ }^{\circ} \mathrm{C}$; ${ }^{1} \mathrm{H}$ NMR $(500 \mathrm{MHz}$, $\left.\mathrm{CDCl}_{3}\right) \delta: 8.93 \sim 8.89(\mathrm{~m}, 1 \mathrm{H}, \mathrm{ArH}), 8.48 \sim 8.43(\mathrm{~m}, 1 \mathrm{H}$, ArH), 8.40 (d, $J=7.9 \mathrm{~Hz}, 1 \mathrm{H}, \operatorname{ArH}), 8.13$ (s, 1H, triazole-H), 7.70 (t, $J=8.0 \mathrm{~Hz}, 1 \mathrm{H}, \mathrm{ArH}), 7.67$ (s, $1 \mathrm{H}$, triazole-H), $7.49 \sim 7.39$ (m, 7H, ArH), $7.09(\mathrm{~s}, 1 \mathrm{H}, \mathrm{CH}), 6.99$ $(\mathrm{s}, 1 \mathrm{H},=\mathrm{CH}), 6.98(\mathrm{~d}, J=8.3 \mathrm{~Hz}, 2 \mathrm{H}, \mathrm{ArH}) ;{ }^{13} \mathrm{C} \mathrm{NMR}$ $\left(400 \mathrm{MHz}, \mathrm{CDCl}_{3}\right) \delta$ : 162.97, 152.74, 148.34, 144.46, $135.53,135.42,134.96,131.08,129.94,129.47,129.13$, $128.84,128.50,127.98,127.46,126.89,125.80,125.77$, 124.71, 76.81. HRMS (ESI) calcd for $\mathrm{C}_{25} \mathrm{H}_{18} \mathrm{~F}_{3} \mathrm{~N}_{4} \mathrm{O}_{4}[\mathrm{M}+$ $\mathrm{H}]^{+}$495.1275, found 495.1271.

(E)-1-Phenyl-2-(1H-1,2,4-triazol-1-yl)-3-(4-(trifluoromethyl)phenyl)allyl 2,3-dichlorobenzoate $(\mathbf{5 j})$ : Yellow solid, yield $33.25 \%$. m.p. $103 \sim 105{ }^{\circ} \mathrm{C} ;{ }^{1} \mathrm{H}$ NMR $(500$ $\left.\mathrm{MHz}, \mathrm{CDCl}_{3}\right) \delta: 8.11(\mathrm{~s}, 1 \mathrm{H}$, triazole-H), $7.67(\mathrm{dd}, J=7.8$, $1.6 \mathrm{~Hz}, 1 \mathrm{H}, \mathrm{ArH}), 7.64$ (s, 1H, triazole-H), 7.47 (d, $J=8.3$ $\mathrm{Hz}, 2 \mathrm{H}, \mathrm{ArH}), 7.45 \sim 7.42(\mathrm{~m}, 2 \mathrm{H}, \mathrm{ArH}), 7.41 \sim 7.36(\mathrm{~m}$, 3H, ArH), $7.31 \sim 7.27$ (m, 2H, ArH), 7.04 (s, 1H, CH), 6.99 $(\mathrm{s}, 1 \mathrm{H},=\mathrm{CH}), 6.95(\mathrm{~d}, J=8.3 \mathrm{~Hz}, 2 \mathrm{H}, \mathrm{ArH}) ;{ }^{13} \mathrm{C} \mathrm{NMR}$ $\left(400 \mathrm{MHz}, \mathrm{CDCl}_{3}\right) \delta$ : 163.71, 152.75, 144.49, 135.66, $135.60,135.04,134.81,133.64,131.91,131.73,131.04$, $130.71,129.95,129.31,128.99,128.80,128.03,127.33$, $127.04,125.75,125.72,124.94,122.24,76.96$. HRMS (ESI) calcd for $\mathrm{C}_{25} \mathrm{H}_{17} \mathrm{Cl}_{2} \mathrm{~F}_{3} \mathrm{~N}_{3} \mathrm{O}_{2}[\mathrm{M}+\mathrm{H}]^{+}$518.0644, found 518.0644 .

\subsection{X-ray crystal structure determination}

The crystal of compound 5a with dimensions of 0.32 $\mathrm{mm} \times 0.28 \mathrm{~mm} \times 0.24 \mathrm{~mm}$ was mounted on a CCD area detector with a graphite-monochromated Mo $\mathrm{K} \alpha$ radiation $(\lambda=0.071073 \mathrm{~nm})$ by using a phi and scan modes at $296(2)$ $\mathrm{K}$ in the range of $4.466^{\circ} \leqslant \theta \leqslant 51.878^{\circ}$. The crystal belongs to monoclinic system with space group $P 2_{1} / n$ and crystal parameters of $a=1.2134(7) \mathrm{nm}, b=1.1142(7) \mathrm{nm}, c=$ $1.6953(14) \mathrm{nm}, \alpha=90^{\circ}, \beta=110.565(18)^{\circ}, \gamma=90^{\circ}, V=$ $2.146(3) \mathrm{nm}^{3}, D_{\mathrm{c}}=1.391 \mathrm{~g} / \mathrm{cm}^{3}$. The absorption coefficient $\mu=0.108 \mathrm{~mm}^{-1}$ and $Z=4$. The structure was solved by direct methods with SHELXS-97 ${ }^{[27]}$ and refined by the full-matrix least squares method on $F^{2}$ data using SHELXL-97. The empirical absorption corrections were applied to all intensity data. The $\mathrm{H}$ atom of $\mathrm{N}-\mathrm{H}$ was initially located in a difference Fourier map and was refined with the restraint $\mathrm{Uiso}(\mathrm{H})=1.2 \mathrm{Ueq}(\mathrm{N})$. Other $\mathrm{H}$ atoms were positioned geometrically and refined using a riding model, with $d(\mathrm{C}-\mathrm{H})=0.093 \sim 0.097 \mathrm{~nm}$ and $\operatorname{Uiso}(\mathrm{H})=$ 


\subsection{Ueq(C) or 1.5 Ueq(Cmethyl).}

\subsection{Fungicidal activity}

The fungicidal activity of compounds $\mathbf{5 a} \sim \mathbf{5} \mathbf{j}$ were tested in vitro against Gibberella zeae (GZ), Phytophthora infestans (PI), Phytophthora capsici (PC), Sclerotinia sclerotiorum (SS), Rhizoctonia solani (RS), Alternaria solani $(A S)$, Botrytis cinerea $(B C)$, Fusarium oxysporum $(F O)$, Cercospora arachidicola (CA), Physalospora piricola $(P P)$ and their relative percent inhibition (\%) has been determined using the mycelium growth rate method ${ }^{[28]}$ and fluxapyroxad was used as positive control. Each compound was dissolved in dimethyl sulfoxide (DMSO) with $1 \%$ tween to prepare the $10000 \mathrm{mg} / \mathrm{L}$ stock solution. The ten fungi were inoculated into a petri dish containing $50 \mu \mathrm{g} / \mathrm{mL}$ stock solution and incubated in a $25{ }^{\circ} \mathrm{C}$ biochemical incubator in dark. The solvent DMSO with $1 \%$ tween was used as blank assay. The fungicidal effect was investigated $3 \mathrm{~d}$ later. Each process is repeated 3 times. The inhibition of compounds compared to the blank assay was calculated via the following equation:

$$
\text { Inhibition }(\%)=(\mathrm{CK}-\mathrm{AI}) / \mathrm{CK} \times 100 \%
$$

where $\mathrm{CK}$ is the average diameter of mycelia in the blank test and AI is the average diameter of mycelia in the presence of those compounds.

\subsection{Docking studies}

Molecular docking between compound 5c and sterol $14 \alpha$ demethylase (CYP51) was done using Discovery Studio 2.5 software. The binding sites were generated from the sterol 14 $\alpha$-demethylase structure (PDB code: $5 J L C)$. The detailed method was done according to our previous work. ${ }^{[29]}$

Supporting Information The ${ }^{1} \mathrm{H}$ NMR, ${ }^{13} \mathrm{C}$ NMR and HRMS spectra of intermediates and target compounds $\mathbf{5 a} \sim$ 5j. Selected crystallographic data and selected molecular structure parameters of compound 5a. The Supporting Information is available free of charge via the Internet at http://sioc-journal.cn.

\section{References}

[1] Yu, C. S.; Wang, Q.; Bajsa-Hirschel, J.; Cantrell, C.; Duke, S. O. Liu, X. H. J. Agric. Food Chem. 2021, 69, 6423.

[2] Almehmadi, M. A.; Aljuhani, A.; Alraqa, S. Y.; Ali, I.; Rezki, N.; Aouad, M. R.; Hagar, M. J. Mol. Struct. 2021, 1225, 129148.

[3] Liu, X. H.; Wen, Y. H.; Cheng, L.; Xu, T. M.; Wu, N. J. J. Agric. Food Chem. 2021, 69, 6968.

[4] Liu, X. H.; Qiao, L.; Zhai, Z. W.; Cai, P. P.; Cantrell, C. L.; Tan, C. X.; Weng, J. Q.; Han, L.; Wu, H. K. Pest Manage. Sci. 2019, 75,
2892.

[5] Liu, X. H.; Weng, J. Q.; Wang, B. L.; Li, Y. H.; Tan, C. X.; Li, Z. M. Res. Chem. Intermed. 2014, 40, 2605.

[6] Liu, X. H.; Xu, X. Y.; Tan, C. X.; Weng, J. Q.; Xin, J. H.; Chen. J. Pest Manage. Sci. 2015, 71, 292.

[7] Kosikowska, U.; Wujec, M.; Trotsko, N.; Plonka, W.; Paneth, P.; Paneth, A. Molecules 2021, 26, 170.

[8] Liu, X. H.; Sun, Z. H.; Yang, M. Y.; Tan, C. X.; Weng, J. Q.; Zhang, Y. G.; Ma, Y. Chem. Biol. Drug Des. 2014, 84, 342.

[9] Cudworth, D. P.; Hegde, V. B.; Yap, M. C. H.; Guenthenspberger, K. A.; Hamilton, C. T.; Pechacek, J. T.; Johnson, P. L.; Bis, S. J.; Tisdell, F. E.; Dripps, J. E.; Bruce, T. J.; Dintenfass, L. P.; Gifford, J. M.; Karr, L. L.; Kempe, M. K.; McCormick, D. C.; Schoonover, J. R. J. Agric. Food Chem. 2007, 55, 7517.

[10] Fan, C. C.; Jiao, S. L.; Qin, M.; Zou, Z. H. ChemistrySelect 2019, 4, 8593.

[11] Arif, M. N.; Nadeem, H.; Paracha, R. Z.; Khan, A. U.; Imran, M.; Ali, F. Lett. Drug Des. Discovery 2019, 16, 734.

[12] Sravya, G.; Nagarjuna, U.; Padmavathi, V.; Rajitha, G.; Priya, S. C.; Padmaja, A. Lett. Drug Des. Discovery 2019, 16, 1233.

[13] Chen, Y.; Li, P.; Su, S. J.; Chen, M.; He, J.; Liu, L. W.; He, M.; Wang, H.; Xue, W. RSC Adv. 2019, 9, 23045.

[14] Tawfik, S. S.; Farahat, A. A.; El-Sayed, M. A. A.; Tantawy, A. S.; Bagato, O.; Ali, M. A. Lett. Drug Des. Discovery 2018, 15, 363.

[15] Nasir, A. F.; Flifl, I. A. Indian J. Heterocycl. Chem. 2020, 30, 453.

[16] Yildirim, N. Lett. Drug Des. Discovery 2020, 17, 954.

[17] Song, M. X.; Yan, R.; Zhang, Y. H.; Guo, D. F.; Zhou, N. M.; Deng, X. Q. J. Enzyme Med. Chem. 2020, 35, 1310.

[18] Abuelhassan, A. H.; Badran, M. M; Hassan, H. A.; Abdelhamed, D.; Elnabtity, S.; Aly, O. M. Med. Chem. Res. 2018, 27, 928.

[19] Yu, W.; Wang, H.; Min, L. J.; Hua, X. W.; Liu, X. H. Chin. J. Org. Chem. 2021, 41, 826 (in Chinese). (余玮，王翰，闵莉静，华学文，刘幸海，有机化学，2021，41， 826.)

[20] Muller, K.; Faeh, C.; Diederich, F. Science 2007, 317, 1881.

[21] Purser, S.; Moore, P. R.; Swallow, S.; Gouverneur, V. Chem. Soc. Rev. 2008, 37, 320

[22] Liu, X. H.; Fang, Y. M.; Xie, F.; Zhang, R. R.; Shen, Z. H.; Tan, C. X.; Weng, J. Q.; Xu, T. M.; Huang, H. Y. Pest Manage. Sci. 2017, 73,1900 .

[23] Kosikowska, U.; Wujec, M.; Trotsko, N.; Plonka, W.; Paneth, P.; Paneth, A. Molecules 2021, 26, 170.

[24] Wang, H.; Zhai, Z. W.; Shi, X. Y.; Tan, C. X.; Weng, J. Q.; Han, L.; Li, B. J.; Liu, X. H. Lett. Drug Des. Discovery 2019, 16, 785.

[25] Zhang, P. P.; Wang, Q.; Min, L. J.; Wu, H. K.; Weng, J. Q.; Tan, C. X.; Zhang, Y. G.; Liu, X. H. J. Mol. Struct. 2020, 1205, 127485.

[26] Oh, K.; Nakai, K.; Yamada, K.; Yoshizada, Y. J. Pestic. Sci. 2012, 37,80 .

[27] Liu, X. H.; Yu, W.; Min, L. J.; Wedge, D. E.; Tan, C. X.; Weng, J. Q.; Wu, H. K.; Cantrell, C. L.; Bajsa-Hischel, J.; Hua, X. W.; Duke, S. O. J. Agric. Food Chem. 2020, 68, 7324.

[28] Hua, X. W.; Liu, W. R.; Su, Y. Y.; Liu, X. H.; Liu, J. B.; Liu, N. N.; Wang, G. Q.; Jiao, X. Q.; Fan, X. Y.; Xue, C. M.; Liu, Y.; Liu, M. Pest Manage. Sci. 2020, 76, 2368.

[29] Fu, Q.; Cai, P. P.; Cheng, L.; Zhong, L. K.; Tan, C. X.; Shen, Z. H.; Han, L.; Liu, X. H. Pest Manage. Sci. 2020, 76, 868. 